\title{
Candidemia: Evolution of Drug Resistance and Novel Therapeutic Approaches
}

\author{
Anna Maria Tortorano' \\ Anna Prigitano' \\ Gianluca Morroni ${ }^{2}$ \\ Lucia Brescini ${ }^{2,3}$ \\ Francesco Barchiesi ${ }^{2,4}$ \\ 'Department of Biomedical Sciences for \\ Health, Università degli Studi di Milano, \\ Milano, Italy; ${ }^{2}$ Department of Biomedical \\ Sciences and Public Health, Università \\ Politecnica delle Marche, Ancona, Italy; \\ ${ }^{3}$ Clinic of Infectious Diseases, Azienda \\ Ospedaliero Universitaria, Ospedali \\ Riuniti Umberto I-Lancisi-Salesi, Ancona, \\ Italy; ${ }^{4}$ Clinic of Infectious Diseases, \\ Azienda Ospedaliera Ospedali Riuniti \\ Marche Nord, Pesaro, Italy
}

\begin{abstract}
Candidemia and invasive candidiasis are the most common healthcare-associated invasive fungal infections, with a crude mortality rate of $25-50 \%$. Candida albicans remains the most frequent etiology, followed by $C$. glabrata, C. parapsilosis and C. tropicalis. With the exception of a limited number of species (ie: C. krusei, C. glabrata and rare Candida species), resistance to fluconazole and other triazoles are quite uncommon. However, recently fluconazoleresistant $C$. parapsilosis, echinocandin-resistant $C$. glabrata and the multidrug resistant $C$. auris have emerged. Resistance to amphotericin $B$ is even more rare due to the reduced fitness of resistant isolates. The mechanisms of antifungal resistance in Candida (altered drug-target interactions, reduced cellular drug concentrations, and physical barriers associated with biofilms) are analyzed. The choice of the antifungal therapy for candidemia must take into account several factors such as type of patient, presence of devices, severity of illness, recent exposure to antifungals, local epidemiology, organs involvement, and Candida species. The first-line therapy in nonneutropenic critical patient is an echinocandin switching to fluconazole in clinically stable patients with negative blood cultures and azole susceptible isolate. Similarly, an echinocandin is the drug of choice also in neutropenic patients. The treatment duration is 14 days after the first negative blood culture or longer in cases of organ involvement. An early removal of vascular catheter improves the outcome. The promising results of new antifungal molecules, such as the terpenoid derivative ibrexafungerp, the novel echinocandin with an enhanced half-life rezafungin, oteseconazole and fosmanogepix, representative of new classes of antifungals, are discussed.
\end{abstract}

Keywords: candidemia, Candida, antifungal resistance, management of candidemia, novel antifungals

\section{Introduction}

Candidemia and invasive candidiasis are the most common healthcare-associated invasive fungal infections. The incidence varies with geographical region and local epidemiology. Analysis of large multicentric surveys reports an overall pooled incidence rate of 3.88 per 100,000 inhabitants per year (range 1.0 to 10.4) with an increasing trend from the 1990s (median 2.18) to the following periods (median 4.67 in the year 2000-2010 and 3.22 in the last decade). ${ }^{1}$ This trend may be justified by a progressive increase in high-risk population related to the prolonged survival of critically ill patients and aging population.

Most of the episodes are hospital-acquired with a reported incidence of 0.17-2.7 episodes per 1000 discharges, $0.30-4.9$ per 10,000 patient days. ${ }^{2}$ However, communityacquired candidemia is emerging as a consequence of an increasing use of long-term intravenous devices such as tunneled intravascular and peripherally inserted central catheter (PICC). ${ }^{3}$
Correspondence: Anna Maria Tortorano Department of Biomedical Sciences for Health, Università degli Studi di Milano, Via Pascal 36, Milano, 20133, Italy Tel +390250315145

$\mathrm{Fax}+390250315146$

Email annamaria.tortorano@unimi.it 
Despite treatment, mortality remains high: crude mortality rate varied from $25 \%$ to $50 \%$, while the attributable mortality rate is estimated around $10-20 \%{ }^{1,3}$

Candidemia has a significant economic burden due to the prolonged stay in hospital, mainly in intensive care unit, and the use of expensive antifungal therapy. A mean total cost per patient with candidemia and invasive candidiasis ranging from $\$ 48,487$ to $\$ 157,574$ was reported in a systematic review including five studies. ${ }^{4}$

\section{Organisms}

Candida albicans is the most frequent cause of candidemia and invasive infections and, although it remains the most common pathogen overall causing these infections, the prevalence of other species has been increasing over time.

The SENTRY program analyzing 20,788 invasive isolates collected from 135 medical centers in 39 countries noted a progressive decrease of the frequency of C. albicans from $57.4 \%$ in $1997-2001$ to $46.4 \%$ in 2015-2016 and a parallel increase in C. glabrata, C. parapsilosis, C.tropicalis, C. krusei and other more rare species. ${ }^{5}$

The increase of $C$. glabrata infections may be associated with an increased fluconazole use for treatment and prophylaxis (this species is frequently isolated from hematological patients receiving fluconazole prophylaxis) or/and with the trend toward increasingly older hospitalized patients whose alimentary tract is frequently colonized by this species.

The species distribution varies by geographic areas with C. glabrata most common in North America (24.3\%) and least common in Latin American region (7.1\%), and C. parapsilosis more common (24.3\%) in Latin America. ${ }^{5}$ Mediterranean countries have higher relative incidences of C. parapsilosis and less C. glabrata and C. albicans than North or Central European countries. The species distribution in Asia shows a high incidence of $C$. tropicalis that in China ranks three accounting for $18.7 \%$ of the Candida bloodstream isolates after C. albicans (32.9\%) and C. parapsilosis $(27.1 \%)$ and in some reports from India it is the most prevalent isolated species. ${ }^{5}$

The relative frequency of the Candida species also depends on the different patient populations considered (ICU, medical, surgical wards, hematology, neonatology).

With the exception of a limited number of species (such as C. krusei, C. glabrata and rare species) azole and echinocandin resistance is quite uncommon and, when it occurs, often develops after long-term use of antifungals for treatment or prophylaxis. Acquisition of amphotericin B resistance is even more rare due to the reduced fitness of resistant isolates. ${ }^{6}$ Although flucytosine presents excellent activity against most Candida species, high rates of acquired resistance to this drug are frequently observed during monotherapy.

In large-scale surveillance studies of bloodstream isolates, the overall prevalence of azole and echinocandin resistance in C. albicans is less than $1 \%$.

Fluconazole resistance in C. glabrata is not uncommon, ranging from $5.6 \%$ to $15.7 \%{ }^{7}$ High rates of fluconazole-resistant $C$. glabrata have been reported both from sentinel and population-based surveillance studies conducted in the USA, Australia, Denmark and Belgium. ${ }^{8}$ Cross-resistance between fluconazole and voriconazole was complete for C. glabrata isolates ( $0 \%$ susceptible to voriconazole among fluconazole-resistant strains). ${ }^{5}$

The haploid genome of this species favors the development of tolerance and resistance to azoles.

Also, echinocandin resistance occurs more frequently in C. glabrata (1.7-3.5\%) than in other Candida species $(0-0.7 \%)$ and this can be due to both the haploid genome and preferential use of echinocandins for treatment of these infections. ${ }^{5,7}$ The increasing azole resistance in C. glabrata encouraged the use of echinocandins for the treatment of infections caused by this species and this provoked a selective pressure for echinocandin resistance. ${ }^{9}$ No echinocandin resistance was present in a collection of isolates from 2001 to 2004, while, starting from 2006 with the growing use of these antifungals, echinocandin resistance was present in $8-11 \%$ of fluconazole resistant $C$. glabrata bloodstream isolates. ${ }^{5,9}$

The presence, albeit limited, of multidrug-resistant C. glabrata isolates is worrying.

The introduction use of matrix-assisted laser desorption ionization-time-of-flight mass spectrometry (MALDITOF) and molecular methods allowed to identify two cryptic species of $C$. glabrata ( $C$. nivariensis and C. bracarensis) that are azole-resistant, but very susceptible to echinocandins. ${ }^{5}$

C. parapsilosis is a species complex of three cryptic species identified by MALDI-TOF or molecular methods: C. parapsilosis sensu stricto that is the most prevalent cause of fungemia, and two less common species C. orthopsilosis and C. metapsilosis.

The lower mortality rate seen with C. parapsilosis is consistent with its reduced virulence relative to C. albicans. ${ }^{10}$ C. parapsilosis is a skin colonizer and it is able to colonize intravascular catheters and rapidly grows 
in high-glucose containing parenteral nutrition administered to patients.

The ability of adherence to intravenous catheters and medical devices and of colonizing the hands of healthcare workers may contribute to invasive infections and clonal outbreaks. C. parapsilosis poses a serious threat to newborns, especially those born prematurely and with low birth weight. The prolonged use of total parenteral nutrition and the transition from the hands of healthcare workers are considered the origin of neonatal infections.

Fluconazole resistance was generally considered to be uncommon among $C$. parapsilosis isolates, however recent reports from different parts of the world suggest that fluconazole resistance in $C$. parapsilosis may emerge following drug pressure of fluconazole treatment and prophylaxis, with subsequent patient-to-patient transmission within the hospital environment. ${ }^{8}$ Cross-resistance to azoles was also described: in the SENTRY surveillance study, $67.3 \%$ of the fluconazole-resistant isolates were also voriconazole. $^{5}$

C. parapsilosis is intrinsically less susceptible to echinocandins because of naturally occurring FKS1 mutations. However, even if $C$. parapsilosis consistently displays higher MIC values compared with other Candida species, an equal outcome was observed in clinical trials. ${ }^{11,12}$

Clonal spreading of echinocandin-resistant C. parapsilosis by the hands of healthcare workers has been reported. ${ }^{13}$ The ability to form tenacious biofilms on vascular catheters and other medically implanted devices is responsible to the resistance to azoles.

C. tropicalis occurs particularly in patients with cancer, chronic liver disease, and hematological malignancies. Resistance to fluconazole occurs in $1.1 \%$ of the isolates of this species up to $37.8 \%$ in Asia Pacific area, while echinocandin resistance $(0.5-0.7 \%)$ is reported only in North and Latin America isolates. ${ }^{5,14}$

Infections by $C$. krusei are rare but characterized by poor response to standard antifungal therapy and a high mortality rate $(40-58 \%)$. This species is inherently resistant to fluconazole, therefore this azole should never be used. On the contrary $95 \%$ of the isolates were susceptible to voriconazole. ${ }^{5}$

In the last years, the selective pressure of prophylaxis contributed to the emergence of less common multiresistant yeast pathogens, most of them identified using MALDI-TOF or sequence-based methods.

Due to the rare number of studied isolates species, specific breakpoints do not exist; however, elevated fluconazole and echinocandin MIC values (MIC50/90, $>4 \mathrm{mg} / \mathrm{L}$ ) were observed for isolates of C. fermentati, C. guilliermondii, C. lipolytica, whereas azole-resistant species $C$. norvegensis and $C$. inconspicua as well as C. dubliniensis, C. kefyr, and C. pelliculosa were very susceptible to echinocandins. ${ }^{5}$

C. palmioleophila, often misidentified as C. guilliermondii or C. famata, is an emerging pathogen in Denmark. Isolates of this species, differently from C. guilliermondii, are highly susceptible to echinocandins and less susceptible to posaconazole and voriconazole and resistant to fluconazole. ${ }^{15}$

C. lusitaniae shows low azole MIC values and elevated echinocandin MICs. In addition, this species, despite the low amphotericin B MIC value in the initial isolates, should be regarded as a poor target for amphotericin $\mathrm{B}$ as resistance mutation arises spontaneously. ${ }^{15}$

Among these rare species, $C$. auris has raised considerable concern because of the fast global spread and some peculiar characteristics. This yeast is able to colonize inert materials and can persist for weeks on surfaces in healthcare environments, leading to high transmissibility and protracted outbreaks. Therefore, isolation of patients, wearing of protective clothing by healthcare workers, screening of patients of the affected wards, skin decontamination with chlorhexidine, and daily and terminal cleaning and disinfection of the patient care environment with effective products (hydrogen peroxide, alcohol-quaternary ammonium compounds, and chlorine-based products) is essential. ${ }^{16,17}$

C. auris can be resistant to any or all the systemic antifungal drugs available.

A systematic review reported a fluconazole resistance rate of $44.3 \%$; a wide range was observed depending on the geographic area, from $15.4 \%$ in Japan to $90 \%$ in India. ${ }^{18-20}$

Also, variable voriconazole susceptibility patterns are reported according to the different clades that are correlated to geographic origin. Variable levels of resistance to the other triazole antifungals, to candins $(0-7 \%)$ and amphotericin B (8-35\%) do not seem to have significant clade-specific differences. ${ }^{20,21}$ Resistance to all three classes of commonly prescribed antifungal drugs (panresistance) has been reported from multiple countries.

\section{Mechanisms of Antifungal Resistance}

Candida antifungal resistance may be primary or secondary depending on the species. Intrinsic, or primary, 
resistance is a characteristic of all isolates of the species, without a previous exposure to drugs; a typical example is the resistance of $C$. krusei to fluconazole. Secondary, or acquired, resistance develops in susceptible isolates as a consequence of the exposure to drugs, usually prolonged treatment or prophylaxis.

Up to now, there are only four classes of antifungals available for the treatment of systemic fungal infections: azoles and polyenes, acting at level of the fungal membrane, echinocandins, acting on the fungal wall, and flucytosine interacting with nuclear acid synthesis. Figure 1 displays the mechanisms of action (Figure 1A) and the mechanisms of resistance (Figure 1B) of the different classes of antifungals.

Azoles act on the biosynthesis of ergosterol by inhibiting the enzyme lanosterol $14-\alpha$-sterol demethylase, leading to the accumulation of toxic sterols in the membrane and consequently to the alteration of the function of the membrane. ${ }^{22,23}$ Azoles have a fungistatic activity against Candida, as well as other yeasts, and this characteristic together with the wide use of these antifungals as prophylaxis has led to the widespread resistance to azoles. ${ }^{24}$ One mechanism associated with azole resistance is the reduction of the drug concentration in the fungal cell caused by the activation of efflux pumps, encoded by the $C D R$ genes of the ATP-binding cassette superfamily and by the $M D R$ genes. The induction of efflux pumps encoded by $C D R$ genes confers resistance to all azoles, on the contrary the induction encoded by of the MDR genes seems to lead only to fluconazole resistance. ${ }^{22}$ Another way in which Candida develops resistance to azoles is the alteration or up-regulation of the gene encoding the azole target enzyme, the ERG11 gene for Candida. Mutations in ERG11 prevent the binding of azoles to the enzymatic site. The intrinsic resistance of C. krusei to fluconazole is attributed to the reduced affinity of ERG11p for this azole. ${ }^{22}$ Furthermore, as an adaptive response to azole exposure, Candida can activate a bypass pathway such as the one based on the mutation in the $E R G 3$ gene that prevents the formation of the toxic sterol 14a-methyl-3,6-diol, allowing normal functionality of the cell membrane. ${ }^{22}$

Echinocandins act on the biosynthesis of $(1,3)-\beta$ D-glucan synthase, encoded by $F K S 1$ and $F K S 2$ genes, preventing the correct synthesis of glucan and leading to the loss of cell wall integrity. Echinocandins have fungicidal activity against the majority of Candida species. Echinocandins resistance or reduced susceptibility are mainly due to mutations in the highly conserved regions of $F K S$ genes; levels of resistance depend on the hot spot mutations and expression level of these genes. ${ }^{22,23,25} \mathrm{An}$ adaptive response to echinocandin treatment is the increased production of other wall components, such as the chitin. ${ }^{22,25}$

The polyenes act by binding directly ergosterol in the membrane, causing the formation of channels through which ions and other cellular components escape, leading to the death of the fungal cell. Amphotericin B has usually a fungicidal activity. Acquired resistance is a consequence of a reduction in ergosterol content in the cell membrane due to alterations in some $E R G$ genes (ERG1, ERG2, ERG3, ERG4, ERG6, ERG11). ${ }^{23}$ For this reason, treatment with an antifungal, such as an azole, that decreases cellular sterol concentrations can lead to polyene resistance. ${ }^{23}$ Acquisition of resistance is extremely rare as mutations that conferred resistance to amphotericin B drastically diminish tolerance to external stresses from the host: amphotericin B-resistant mutants were hypersensitive to oxidative stress, febrile temperatures, and killing by neutrophils and also had defects in filamentation and tissue invasion. ${ }^{6}$

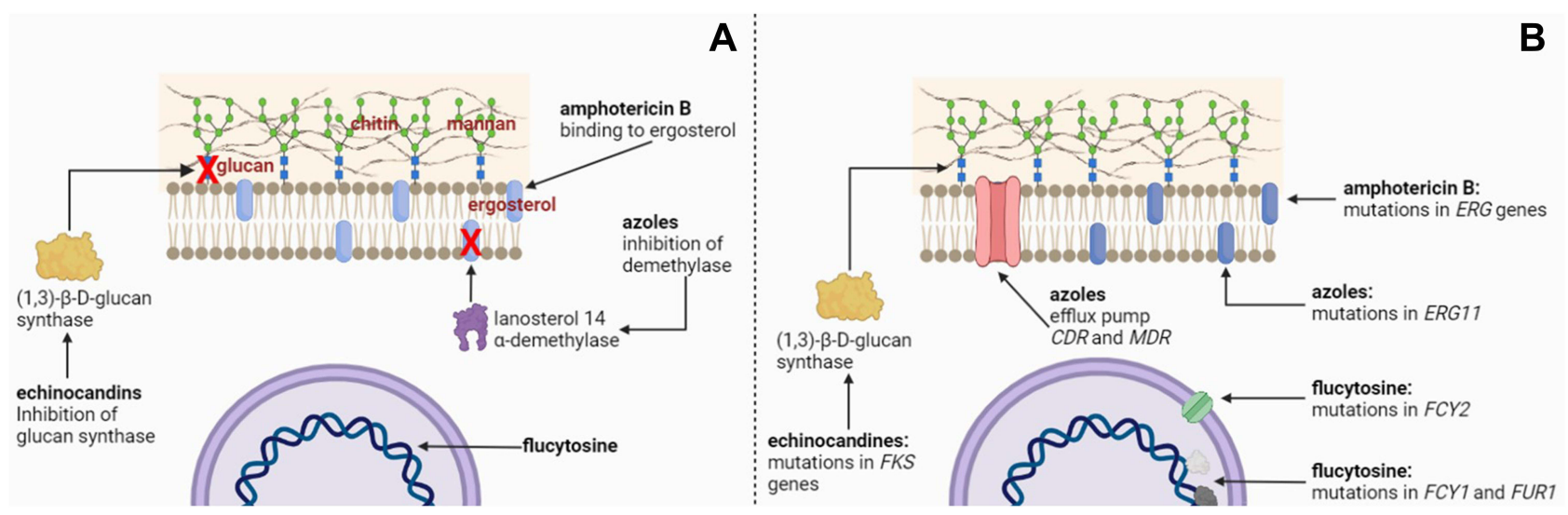

Figure I Antifungals: mechanisms of action (A), mechanisms of resistance (B). Created with BioRender.com. 
Flucytosine (5-fluorocytosine, 5FC) is an antifungal that targets nucleic acid biosynthesis. It is transported into the fungal cell by a permease, encoded by the FCY2 gene, and here is converted into two metabolites, 5-fluorouracil and 5-fluorouridine monophosphate, by the enzymes encoded by the genes FCY1 and FUR1, respectively. The active metabolites inhibit transcription, DNA replication and protein synthesis. Resistance to flucytosine, that emerge when used in monotherapy, have been attributed to mutations in the FCY2, FCY1 and FUR1 genes. ${ }^{26}$ In $C$. glabrata, arginine homeostasis, cell wall remodeling and aquaglyceroporins of the Fps family have emerged as mechanisms of resistance to flucytosine. ${ }^{27}$

Biofilm is another important drug resistance mechanism. The extracellular matrix (ECM) acts as a physical barrier between microorganisms and drug or host immune response and promoting the development of cells able to tolerate high concentrations of antifungals. Other factors can play an important role in conferring resistance to microorganisms organized in the sessile form: alterations in efflux pump expression, changes in cell membrane and wall composition, changes in cellular stress response. ${ }^{24}$ Morphology, characteristics of the ECM, and ability to confer antifungal resistance may differ depending on the Candida species: C. albicans biofilm exhibits a heterogenous structure of blastospores and hyphae in an ECM of polysaccharide material; C. glabrata biofilm is composed by cells in a multilayer structure tightly packed or in clusters of cells; C. tropicalis biofilm is formed by a network of yeasts, pseudohyphae and hyphae, with high germination of hyphae; and $C$. parapsilosis biofilm has clusters of yeast cells adherent to the surface and thin ECM. ${ }^{28}$

Fluconazole, voriconazole and itraconazole fail to eliminate Candida biofilms, whereas echinocandins and amphotericin B lipid formulations are known for their antibiofilm activity, with differences between young and mature biofilms. Studies showed that MICs of liposomal amphotericin B and amphotericin B lipid complex (L-AMB) used against $C$. albicans biofilms are similar to those obtained against planktonic cells. Also echinocandins are active in vitro against Candida biofilms, ${ }^{28-30}$ but differences depending on the species are observed: low MICs for all echinocandins against biofilm of C. albicans and C. krusei, and high MICs for C. lusitaniae and C. guilliermondii, are reported. Micafungin seems more active than the other echinocandins against $C$. parapsilosis biofilm. $^{31}$
Tests in vitro performed on catheters infected separately with $C$. albicans and $C$. glabrata isolates, and treated with micafungin, caspofungin and posaconazole, showed that all three antifungals, especially micafungin, lead to a reduction in Candida biofilms. ${ }^{28}$

The activity of $12 \mathrm{~h} \mathrm{L-AMB}$ locks was equivalent to those of micafungin and caspofungin against biofilm of C. albicans and C. glabrata, but less efficient against C. parapsilosis mature biofilms. However, overall eradication of the biofilm from the catheter was never obtained with any antifungal. ${ }^{32}$

Multidrug resistance (MDR) was defined by the US Centers for Disease Control and Prevention as acquired nonsusceptibility to $\geq 1$ agent in at least 3 antimicrobial categories, but since only fluconazole and echinocandins are recommended as first-line agents for invasive candidiasis, MDR Candida can be defined as an isolate nonsusceptible to $\geq 1$ agent in $\geq 2$ drug classes. $^{26}$ The development of MDR Candida, although rare compared to antibacterials, is a matter of concern especially in light of the changing epidemiology of Candida infections, showing a shift towards species intrinsically resistant to the most commonly used antifungal drugs. In fact, MDR Candida mainly involves acquired resistance in species with intrinsic resistance ${ }^{26}$ such as echinocandin resistance in C. krusei, C. glabrata, C. guillermondii or C. auris. MDR occurs more rarely in species without inherent resistance as this requires the acquisition of different resistance mechanisms which have a fitness cost; for instance, C. albicans has been reported to acquire MDR after antifungal exposure in the setting of longterm echinocandin use. $^{26}$

Of particular concern are the increasingly reported cases of MDR of C. glabrata and C. parapsilosis.

\section{Management of Candidemia}

The choice of antifungal therapy for the treatment of candidemia must take into account several variables such as type of patient (neutropenic or non-neutropenic patient), presence of acute and chronic comorbidities, presence of devices (urinary or central vascular catheter), severity of illness, recent exposure to antifungal agents (azole or echinocandin), local epidemiology, organs involvement, Candida species (Table 1). ${ }^{33}$ In the previous literature, the rate of mortality appears to be related to the presence of certain risk factors such as age, higher APACHE score, immunosuppression, renal failure, triazole exposure in both neutropenic and non-neutropenic patients. ${ }^{34-36}$ On 
Table I Clinical Conditions/Risk Factors Associated with Candidemia, Resistance Rates, Antifungal Treatment According to Different Candida Species

\begin{tabular}{|c|c|c|c|}
\hline Candida spp. & $\begin{array}{l}\text { Patients at Risk/Risk } \\
\text { Factors }\end{array}$ & Rate of Resistance & Therapy \\
\hline C. albicans & All patients & $\begin{array}{l}\text { Fluconazole: } 0.1-0.4 \% \\
\text { Echinocandins: } 0-0.1 \% \\
\text { Amphotericin B: rare }\end{array}$ & $\begin{array}{l}\text { - Echinocandins (I) } \\
\text { - Fluconazole, } 800 \mathrm{mg} \text { then } 400 \mathrm{mg}(2) \\
\text { - Liposomal amphotericin B, 3-5 mg/kg/day (3) }\end{array}$ \\
\hline C. parapsilosis & $\begin{array}{l}\text { ICU patients } \\
\text { Neonates } \\
\text { Vascular catheter }\end{array}$ & $\begin{array}{l}\text { Fluconazole: } 0.6 \text { up to } 53 \% \\
\text { Echinocandins: } 0-0.1 \% \\
\text { Amphotericin B: rare }\end{array}$ & $\begin{array}{l}\text { - Echinocandins (I) } \\
\text { - Fluconazole, } 800 \mathrm{mg} \text { then } 400 \mathrm{mg}(2)\end{array}$ \\
\hline C. glabrata & $\begin{array}{l}\text { Older age } \\
\text { Diabetes } \\
\text { Cancer } \\
\text { Hematological malignancies } \\
\text { Stem cell transplantation } \\
\text { Azole prophylaxis }\end{array}$ & $\begin{array}{l}\text { Fluconazole: } 2.6-10.6 \% \\
\text { Echinocandins: } 0 \%-2.8 \% \\
\text { Amphotericin B: rare }\end{array}$ & $\begin{array}{l}\text { Fluconazole and voriconazole are not recommended } \\
\text { for frequent azoles resistance } \\
\text { - Echinocandins (I) } \\
\text { - Liposomal amphotericin B, 3-5 mg/kg/day (3) }\end{array}$ \\
\hline C. tropicalis & $\begin{array}{l}\text { Corticosteroid therapy } \\
\text { Hematological malignancies } \\
\text { Stem cell transplantation }\end{array}$ & $\begin{array}{l}\text { Fluconazole: } 1.1-37.8 \% \\
\text { Echinocandins: } 0-1.3 \% \\
\text { Amphotericin B: rare }\end{array}$ & $\begin{array}{l}\text { - Echinocandins (I) } \\
\text { - Fluconazole, } 800 \mathrm{mg} \text { then } 400 \mathrm{mg}(2) \\
\text { - Liposomal amphotericin B, 3-5 mg/kg/day (3) }\end{array}$ \\
\hline C. krusei & $\begin{array}{l}\text { Corticosteroid therapy } \\
\text { Hematological malignancies } \\
\text { Stem cell transplantation } \\
\text { Azole prophylaxis }\end{array}$ & $\begin{array}{l}\text { Fluconazole: innately } \\
\text { Echinocandins: } 0-0.7 \% \\
\text { Amphotericin B: rare }\end{array}$ & $\begin{array}{l}\text { Fluconazole is not recommended for frequent azoles } \\
\text { resistance } \\
\text { - Echinocandins (I) } \\
\text { - Liposomal amphotericin B, 3-5 mg/kg/day (3) } \\
\text { - Voriconazole (4) }\end{array}$ \\
\hline C. auris & $\begin{array}{l}\text { Diabetes } \\
\text { Cancer } \\
\text { Hematological malignancies } \\
\text { ICU patients } \\
\text { Invasive procedures }\end{array}$ & $\begin{array}{l}\text { Fluconazole: } 15.4-90 \% \\
\text { Voriconazole: } 50 \% \\
\text { Echinocandins: } 2-8 \% \\
\text { Amphotericin B: } 15-30 \%\end{array}$ & - Echinocandins (I) \\
\hline
\end{tabular}

Notes: (I) Caspofungin: loading dose $70 \mathrm{mg}$, then $50 \mathrm{mg}$ daily; micafungin: $100 \mathrm{mg}$ daily; anidulafungin: loading dose $200 \mathrm{mg}$, then $100 \mathrm{mg}$ daily. (2) In stable patients without previous exposure to azoles. (3) If isolates are not susceptible to azoles and echinocandins or in the presence of organ involvement. (4) $6 \mathrm{mg} / \mathrm{kg} \mathrm{q} / 2 \mathrm{~h} \times 2$ doses (load) then $3-4 \mathrm{mg} / \mathrm{kg} \mathrm{q} / 2 \mathrm{~h}$.

the contrary, early initiation of antifungal therapy and adequate control of the source of infection are factors that reduce mortality in patient with candidemia. ${ }^{37,38}$

The Infectious Diseases Society of America (IDSA) and European Society of Clinical Microbiology and Infectious Diseases (ESCMID) guidelines for the management of candidemia recommend performing transesophageal echocardiography and fundoscopy. Data from the literature showed a percentage of endocarditis equal to $8.3 \%{ }^{39}$ and a percentage of ocular involvement equal to $16 \%$ in patients with candidemia. ${ }^{40}$

The presence of organ involvement affects the choice of the type of antifungal to be administered, in relation to their penetration in the different districts, and the duration of the antifungal therapy. ${ }^{33,41}$
The antimycogram of Candida isolates should be always performed for a correct selection of the antifungal agent. $^{33}$ However, even in the presence of an in vitro susceptible strain, there could be clinical resistance linked to the pharmacokinetics and pharmacodynamics of the antifungal drug.

\section{Non-Neutropenic Patients}

The first-line therapy in the non-neutropenic critical patient is an echinocandin (caspofungin: loading dose $70 \mathrm{mg}$, then $50 \mathrm{mg}$ daily; micafungin: $100 \mathrm{mg}$ daily; anidulafungin: loading dose $200 \mathrm{mg}$, then $100 \mathrm{mg}$ daily), according to the IDSA and European Association for the Study of the Liver (EASL) guidelines. ${ }^{33,41}$ Fluconazole, intravenous or oral, $800 \mathrm{mg}(12 \mathrm{mg} / \mathrm{kg})$ loading dose, then 
$400 \mathrm{mg}(6 \mathrm{mg} / \mathrm{kg})$ daily, can be administered in clinically stable patients, who have not been exposed to azoles and are not at risk for C. glabrata infection. ${ }^{33}$ Furthermore, these guidelines recommend step down strategy in patients in therapy with echinocandin who are clinically stable with negative blood cultures and Candida isolate susceptible to azoles, switching to fluconazole (for C. albicans, C. parapsilosis and C. tropicalis) or voriconazole (for C. krusei) within 10 days. $^{33,41,42}$ All three echinocandins demonstrated a broad spectrum, fungicidal activity, antibiofilm activity, favorable safety profile and limited drug interactions. $^{33,41,43}$ However, these drugs do not reach therapeutic concentrations in the eye, urine and central nervous system. ${ }^{42}$ No differences in efficacy and tolerability between echinocandins were reported in the literature, however only anidulafungin has been compared with fluconazole. ${ }^{44}$ This clinical trial showed that patients with candidemia treated with anidulafungin had a better outcome than those treated with fluconazole. This finding was also confirmed in a subsequent sub-analysis carried out by the same authors, in which anidulafungin showed greater efficacy with respect to fluconazole, especially in C. albicans infections, ${ }^{45}$ and in another retrospective analysis performed in ICU patients. ${ }^{46}$ In particular, this secondary analysis showed, a global response rate equal to $70.8 \%$ for anidulafungin and $54.1 \%$ for fluconazole, allcause mortality equal to $10.1 \%$ versus $20.3 \%$ at 14 days and $20.2 \%$ versus $24.3 \%$ at 28 days for anidulafungin and fluconazole, respectively.

Furthermore, acquired resistance for echinocandins in Candida is rare. However, over the years, there has been an increase in resistance to echinocandins, especially in C. glabrata. ${ }^{47,48}$ Probably, the greater use of these drugs favored the acquisition of resistance mechanisms. For this reason, de-escalation to fluconazole or voriconazole is recommended in stable patients with Candida susceptible species. In previous literature, is also reported less in vitro activity of echinocandins in C. parapsilosis, ${ }^{47,48}$ but no clinical studies have demonstrated the superiority of fluconazole or other antifungal therapy in the treatment of C. parapsilosis infections. ${ }^{49-51}$

Voriconazole represents a therapeutic alternative in fluconazole-resistant isolates of C. krusei, C. guilliermondii, C. glabrata for transition from an echinocandin or amphotericin B to oral therapy. ${ }^{33}$ Respect to other antifungal drugs, voriconazole presented multiple drug-drug interactions, variable pharmacokinetics, and renal toxicity in case of parenteral formulation. ${ }^{33,41}$
Amphotericin B has a broad-spectrum activity, except for C. lusitaniae. Lipid formulation of amphotericin B (3 mg/kg daily) is considered in patients with intolerance or absence of clinical and microbiological response to echinocandins and/or azoles and in patients with suspicion of other fungal infections. ${ }^{33}$

The duration of treatment recommended by the guidelines in uncomplicated candidemia is 14 days after the first negative blood culture, but it is longer in cases with organ involvement.

The antifungal regimen of choice in Candida endocarditis is liposomal amphotericin B and the surgery is recommended. ${ }^{41}$ In ocular candidiasis, fluconazole or voriconazole must be used, if the isolates are susceptible, and liposomal amphotericin B alone or combined with flucytosine, when the susceptibility is unknown due to the better penetration in this district. ${ }^{41}$

In non-neutropenic patients, candidemia is very frequently associated with the presence of central vascular catheter (CVC) ( $70 \%$ of cases) and several retrospective studies have demonstrated a better outcome and a shorter duration of candidemia in patients with early CVC removal. ${ }^{33}$ The IDSA guidelines recommended that earlier CVC removal is possible when the source of infection is the CVC, individualizing this decision for each patient. If the catheter removal is not possible, echinocandin or amphotericin B is preferable to fluconazole, for their greater penetration into the biofilm. ${ }^{41}$

\section{Neutropenic Patients}

In neutropenic patients, treatment of fungal infection plays a crucial role in improving survival. For invasive candidiasis and candidemia, therapy should be administered for 14 days after the last positive blood. ${ }^{36}$ In these cases, echinocandins are the antifungal therapy of choice, with no difference between anidulafungin, caspofungin or micafungin. The overall recommendation assigned a lower score to anidulafungin due to the low number of neutropenic patients in the clinical trial. ${ }^{44}$ Also, liposomal formulation of amphotericin B could be used but is less recommended due to the potential toxicity. ${ }^{51}$ The other formulations of amphotericin $\mathrm{B}$, as the conventional amphotericin B, are excluded due to their nephrotoxicity. ${ }^{52}$ Regarding azoles, only fluconazole received a weak recommendation in infections sustained by susceptible isolates. Although some trials support the use of this antifungal, they did not take into account the growing rate of resistance. ${ }^{53,54}$ Voriconazole could be 
limitedly used in infection where a mold cover is desirable. For the other azoles, the data are limited and cannot suggest their use. ${ }^{55}$ Furthermore, data on antifungal combination are not conclusive; however, the combination therapy could be helpful in severe and complicated infections. ${ }^{36}$

In some neutropenic patients, chronic disseminated candidiasis (or hepato-splenic candidiasis) is a disease that usually occurred after chemotherapy. Data on these infections are limited, but there are some recommendations similar to those of the invasive candidiasis. Lipid formulations of amphotericin $\mathrm{B}$ alongside the use of an echinocandin are encouraged, followed by fluconazole in infections with susceptible isolates. ${ }^{33}$ Unlike candidemia, the therapy should be prolonged for at least 8 weeks or until the splenic lesion is resolved. ${ }^{33,36}$

In infections related to CVCs, the removal of the catheter seems to have a crucial role (specially in infections sustained by $C$. parapsilosis) unless an echinocandin was used for the treatment. In the latter case, the studies reported a favorable outcome even though the numbers are low. ${ }^{50,51,56}$

\section{New Antifungal Agents}

Antifungal resistance in Candida is growing in the last years and underlines the necessity of new antifungal to treat multi-drug resistant isolates. Although the last class of antifungal agent was licensed more than 20 years ago, to date there are some new antifungal agents currently in clinical trials and some of them already in Phase III.

Ibrexafungerp is a novel terpenoid derived from enfumafungin with mechanism of action similar to echinocandins. It binds the 1,3-glucan synthase, but via alternative binding sites rendering it unaffected by FKS mutations. ${ }^{57}$ Differently from echinocandins, ibrexafungerp can be administered both intravenously and orally and could be used in different fungal infections, from superficial to lifethreatening. ${ }^{58}$ Several clinical trials studied the effectiveness of ibrexafungerp in invasive candidiasis, invasive pulmonary aspergillosis and vulvovaginal candidiasis (VVC). The recent Phase III trial VANISH 303 detected higher rate of clinical cure, mycological eradication and overall success of oral ibrexafungerp compared with placebo in VVC. ${ }^{59}$ Moreover, ibrexafungerp is active against clinical isolates of Candida, including strains resistant to echinocandins and other antifungal agents. ${ }^{60,61}$

Rezafungin is a novel echinocandin with an enhanced half-life and a long stability. These features enabled the administration once-weekly instead of the daily dose of the other echinocandins. ${ }^{62}$ Rezafungin showed similar or little less activity compared to other echinocandins, but overall better results than azoles or amphotericin B. ${ }^{63,64}$ In Phase II trial, rezafungin with a weekly dose regimen of $400 \mathrm{mg}$ in the first week followed by a $200 \mathrm{mg}$ administration demonstrated similar effectiveness compared to caspofungin in patients with candidemia or invasive candidiasis. ${ }^{65}$

Representative of a new class of antifungal agents, oteseconazole is a tetrazole that inhibits lanosterol demethylase. In phase II studies, oral formulation of oteseconazole was administered in patients with VVC and demonstrated tolerability and similar effectiveness compared to fluconazole. ${ }^{66,67}$ In vitro, this new antifungal agent showed potent in vitro activity against fluconazoleresistant Candida isolates, though in some strains MICs were high suggesting novel resistance mechanisms. ${ }^{68,69}$

As otesaconazole, also fosmanogepix is a novel drug belonging to a new class of antifungal that completed a Phase 2 clinical trial for candidiasis in non-neutropenic patients (NCT03604705). Fosmanogepix is a prodrug that is converted into the active formulation manogepix by the systemic phosphatase. This drug inhibits Gtw1, an enzyme involved in mannoproteins trafficking and anchoring. ${ }^{70} \mathrm{In}$ vitro, it demonstrated a potent effect against Candida spp. (including echinocandin- and azole-resistant strains) resulting the most active drug compared to anidulafungin, micafungin and fluconazole. ${ }^{71}$ Notably, strains with acquired resistance to fluconazole also showed increased MIC to manogepix though the mechanism of this correlation remains unexplained. ${ }^{72}$

\section{Conclusions}

As the use of antifungals is widespread in modern medicine, resistant fungal infections, including those caused by Candida spp., have been on the rise. While C. albicans isolates display low resistance rates to the most common drugs utilized in clinical practice, other Candida species could represent a problem in terms of clinical management due to the increasingly reported low susceptibility profiles to antifungals. In particular, resistance to azoles and echinocandins among C. glabrata as well as resistance to fluconazole among C. parapsilosis are not rare phenomena nowadays. Additionally, we are facing with an increasing isolation of "new", "MDR" opportunistic pathogens, such as C. auris, for which few available antifungal molecules are active. Hence, at least three priorities should be pursued for a better management of fungal infections. First, appropriate species 
identification methods and antifungal susceptibility testing via standardized procedures are of utmost importance and should be implemented. Second, drugs with unique mechanisms of action able to overcome the resistance mechanisms should be introduced to our current armamentarium. Finally, in order to minimize the selection of resistant strains, an effort must be made to avoid empirical therapy as much as possible.

\section{Disclosure}

AP and AMT received speaker honorarium from Gilead. The other authors report no conflicts of interest in this work.

\section{References}

1. Koehler P, Stecher M, Cornely OA, et al. Morbidity and mortality of candidaemia in Europe: an epidemiologic meta-analysis. Clin Microbiol Infect. 2019;25(10):1200-1212. doi:10.1016/j. cmi.2019.04.024

2. Prigitano A, Cavanna C, Passera M. Evolution of fungemia in an Italian region. $J$ Mycol Med. 2020;30(1):100906. doi:10.1016/j. mycmed.2019.100906

3. Pappas P, Lionakis M, Arendrup M, et al. Invasive candidiasis. Nat Rev Dis Primers. 2018;4(1):18026. doi:10.1038/nrdp.2018.26

4. Wan Ismail WNA, Jasmi N, Khan TM, Hong YH, Neoh CF. The economic burden of candidemia and invasive candidiasis: a systematic review. Value Health Reg Issues. 2020;21:53-58. doi:10.1016/j.vhri.2019.07.002

5. Pfaller MA, Diekema DJ, Turnidge JD, Castanheira M, Jones RN. Twenty years of the SENTRY antifungal surveillance program: results for Candida species from 1997-2016. Open Forum Infect Dis. 2019;6(Suppl 1):S79-S94. doi:10.1093/ofid/ofy358

6. Vincent BM, Lancaster AK, Scherz-Shouval R, Whitesell L, Lindquist $\mathrm{S}$. Fitness trade-offs restrict the evolution of resistance to amphotericin B. PLoS Biol. 2013;11(10):e1001692. doi:10.1371/journal.pbio. 1001692

7. Lockhart SR. Current epidemiology of Candida infection. Clin Microbiol Newsletter. 2014;36(17):131-136. doi:10.1016/j. clinmicnews.2014.08.001

8. Castanheira M, Deshpande LM, Messer SA, Rhomberg PR, Pfaller MA. Analysis of global antifungal surveillance results reveals predominance of Erg11 Y132F alteration among azole-resistant Candida parapsilosis and Candida tropicalis and country-specific isolate dissemination. Int J Antimicrob Agents. 2020;55:105799. doi:10.1016/j.ijantimicag.2019.09.003

9. Pfaller MA, Castanheira M, Lockhart SR, Ahlquist AM, Messer SA, Jones RN. Frequency of decreased susceptibility and resistance to echinocandins among fluconazole-resistant bloodstream isolates of Candida glabrata. J Clin Microbiol. 2012;50(4):1199-1203. doi:10.1128/JCM.06112-11

10. Tóth R, Nosek J, Mora-Montes HM. Candida parapsilosis: from genes to the bedside. Clin Microbiol Rev. 2019;32:e0111-18. doi:10.1128/CMR.00111-18

11. Chiotos K, Vendetti N, Zaoutis TE, et al. Comparative effectiveness of echinocandins versus fluconazole therapy for the treatment of adult candidaemia due to Candida parapsilosis: a retrospective observational cohort study of the Mycoses Study Group (MSG-12). Antimicrob Chemother. 2016;71(12):3536-3539. doi:10.1093/jac/dkw305

12. Arendrup MC, Friberg N, Mares M, Kahlmeter G, Meletiadis J, Guinea J. How to interpret MICs of antifungal compounds according to the revised clinical breakpoints v. 10.0 European committee on antimicrobial susceptibility testing (EUCAST). Clin Microbiol Infect. 2020;26(11):1464-1472. doi:10.1016/j.cmi.2020.06.007
13. Barchiesi F, Caggiano G, Falconi Di Francesco L, Montagna MT, Barbuti S, Scalise G. Outbreak of fungemia due to Candida parapsilosis in a pediatric oncology unit. Diagn Microbiol Infect Dis. 2004;49(4):269-271. doi:10.1016/j.diagmicrobio.2004.03.011

14. Ngamchokwathana C, Chongtrakool P, Waesamaae A, Chayakulkeeree M. Risk factors and outcomes of non-albicans Candida bloodstream infection in patients with candidemia at Siriraj Hospital-Thailand's largest national tertiary referral hospital. J Fungi. 2021;7:269. doi:10.3390/jof7040269

15. Jensen RH, Arendrup MC. Candida palmioleophila: characterization of a previously overlooked pathogen and its unique susceptibility profile in comparison with five related species. J Clin Microbiol. 2011;49(2):549-556. doi:10.1128/JCM.02071-10

16. Chowdhary A, Sharma C, Meis JF. Candida auris: a rapidly emerging cause of hospital-acquired multidrug-resistant fungal infections globally. PLoS Pathog. 2017;13(5):e1006290. doi:10.1371/journal.ppat.1006290

17. Rutala WA, Kanamori H, Gergen MF, Sickbert-Bennett EE, Weber DJ. Susceptibility of Candida auris and Candida albicans to 21 germicides used in healthcare facilities. Infect Control Hosp Epidemiol. 2019;40:380-382. doi:10.1017/ice.2019.1

18. Osei Sekyere J. Candida auris: a systematic review and meta-analysis of current updates on an emerging multidrug-resistant pathogen. Microbiol Open. 2018;7(4):e00578. doi:10.1002/mbo3.578

19. Iguchi S, Itakura Y, Yoshida A, et al. Candida auris: a pathogen difficult to identify, treat, and eradicate and its characteristics in Japanese strains. J Infect Chemother. 2019;25(10):743-749. doi:10.1016/j.jiac.2019.05.034

20. Chowdhary A, Prakash A, Sharma C, et al. A multicentre study of antifungal susceptibility patterns among 350 Candida auris isolates (2009-17) in India: role of the ERG11 and FKS1 genes in azole and echinocandin resistance. $J$ Antimicrob Chemother. 2018;73 (4):891-899. doi:10.1093/jac/dkx480

21. Szekely A, Borman AM, Johnson EM. Candida auris isolates of the Southern Asian and South African lineages exhibit different phenotypic and antifungal susceptibility profiles in vitro. $J$ Clin Microbiol. 2019;57:e02055-18. doi:10.1128/JCM.02055-18

22. Sanguinetti M, Posteraro B, Lass-Flörl C. Antifungal drug resistance among Candida species: mechanisms and clinical impact. Mycoses. 2015;58(Suppl. 2):2-13. doi:10.1111/myc. 12330

23. Perlin DS, Rautemaa-Richardson R, Alastruey-Izquierdo A. The global problem of antifungal resistance: prevalence, mechanisms, and management. Lancet Infect Dis. 2017;17:e383-392. doi:10.1016/ S1473-3099(17)30316-X

24. Robbins N, Caplan T, Cowen LE. Molecular evolution of antifungal drug resistance. Anпu Rev Microbiol. 2017;71:753-775. doi:10.1146/ annurev-micro-030117-020345

25. Revie NM, Iyer KR, Robbins N, Cowen LE. Antifungal drug resistance: evolution, mechanisms and impact. Curr Opin Microbiol. 2018;45:70-76. doi:10.1016/j.mib.2018.02.005

26. Arendrup MC, Patterson TF. Multidrug-Resistant Candida: epidemiology, molecular mechanisms, and treatment. J Infect Dis. 2017;216(S3):S445-451. doi:10.1093/infdis/jix131

27. Costa C, Ponte A, Pais P, et al. New mechanisms of flucytosine resistance in C. glabrata unveiled by a chemogenomics analysis in S. cerevisiae. PLoS One. 2015;10:e135110. doi:10.1371/journal.pone.0135110

28. Cavalheiro M, Teixeira MC. Candida Biofilms: threats, challenges, and promising strategies. Front Med. 2018;5:28. doi:10.3389/ fmed.2018.00028

29. Katragkou A, Kruhlak MJ, Simitsopoulou M, et al. Interactions between human phagocytes and Candida albicans biofilms alone and in combination with antifungal agents. J Infect Dis. 2010;201 (12):1941-1949. doi:10.1086/652783

30. Kaneko Y, Miyagawa S, Takeda O, et al. Real-time microscopic observation of Candida biofilm development and effects due to micafungin and fluconazole. Antimicrob Agents Chemother. 2013;57 (5):2226-2230. doi:10.1128/AAC.02290-12 
31. Simitsopoulou M, Peshkova P, Tasina E, et al. Species-specific and drug-specific differences in susceptibility of Candida biofilms to echinocandins: characterization of less common bloodstream isolates. Antimicrob Agents Chemother. 2013;57(6):2562-2570. doi:10.1128/AAC.02541-12

32. Toulet D, Debarre C, Imbert C. Could liposomal amphotericin B (L-AMB) lock solutions be useful to inhibit Candida spp. biofilms on silicone biomaterials? J Antimicrob Chemother. 2012;67:430-432. doi:10.1093/jac/dkr473

33. Pappas PG, Kauffman CA, Andes DR, et al. Clinical practice guideline for the management of candidiasis: 2016 update by the Infectious Diseases Society of America. Clin Infect Dis. 2016;62(4):409-417. doi:10.1093/cid/civ1194

34. Pfaller M, Neofytos D, Diekema D, et al. Epidemiology and outcomes of candidemia in 3648 patients: data from the Prospective Antifungal Therapy (PATH Alliance) registry, 2004-2008. Diagn Microbiol Infect Dis. 2012;74:323-331. doi:10.1016/j. diagmicrobio.2012.10.003

35. Andes DR, Safdar N, Baddley JW, et al. Impact of treatment strategy on outcomes in patients with candidemia and other forms of invasive candidiasis: a patient-level quantitative review of randomized trials. Clin Infect Dis. 2012;54:1110-1122. doi:10.1093/cid/cis021

36. Ullmann AJ, Akova M, Herbrecht R. ESCMID* guideline for the diagnosis and management of Candida diseases 2012: adults with haematological malignancies and after haematopoietic stem cell transplantation (HCT). Clin Microbiol Infect. 2012;7:53-67. doi:10.1111/1469-0691.12041

37. Kollef M, Micek S, Hampton N, Doherty JA, Kumar A. Septic shock attributed to Candida infection: importance of empiric therapy and source control. Clin Infect Dis. 2012;54:1739-1746. doi:10.1093/cid/ cis 305

38. Ostrosky-Zeichner L, Kullberg BJ, Bow EJ, et al. Early treatment of candidemia in adults: a review. Med Mycol. 2011;49:113-120. doi:10.3109/13693786.2010.512300

39. Fernandez-Cruz A, Menarguez MC, Pedromingo M, et al. Candida endocarditis: yield of echocardiogram in patients with candidemia. 50th Interscience Conference on Antimicrobial Agents and Chemotherapy: Boston, MA; 2010.K-2172.

40. Oude Lashof AM, Sobel JD, Ruhnke M, et al. Safety and tolerability of voriconazole in patients with baseline renal insufficiency and candidemia. Antimicrob Agents Chemother. 2012;56:3133-3137. doi:10.1128/AAC.05841-11

41. Cornely OA, Bassetti M, Calandra T, et al. ESCMID* guideline for the diagnosis and management of Candida diseases 2012: nonneutropenic adult patients. Clin Microbiol Infect. 2012;7:19-37. doi:10.1111/1469-0691.12039

42. Antinori S, Milazzo L, Sollima S, Galli M, Corbellino M. Candidemia and invasive candidiasis in adults: a narrative review. Eur J Intern Med. 2016;34:21-28. doi:10.1016/j.ejim.2016.06.029

43. Maertens J, Theunissen K, Verhoef G, et al. Galactomannan and computed tomography-based preemptive antifungal therapy in neutropenic patients at high risk for invasive fungal infection: a prospective feasibility study. Clin Infect Dis. 2005;41:1242-1250. doi: $10.1086 / 496927$

44. Reboli AC, Rotstein C, Pappas PG, et al. Anidulafungin versus fluconazole for invasive candidiasis. $N$ Engl $J$ Med. 2007;356:2472-2482. doi:10.1056/NEJMoa066906

45. Reboli AC, Shorr AF, Rotstein C, et al. Anidulafungin compared with fluconazole for treatment of candidemia and other forms of invasive candidiasis caused by Candida albicans: a multivariate analysis of factors associated with improved outcome. BMC Infect Dis. 2011;11:261. doi:10.1186/1471-2334-11-261

46. Kett DH, Azoulay E, Echeverria PM, Vincent JL. Candida bloodstream infections in intensive care units: analysis of the extended prevalence of infection in intensive care unit study. Crit Care Med. 2011;39:665-670. doi:10.1097/CCM.0b013e318206c1ca
47. Pfaller MA, Moet GJ, Messer SA, Jones RN, Castanheira M. Candida bloodstream infections: comparison of species distributions and antifungal resistance patterns in community-onset and nosocomial isolates in the SENTRY Antimicrobial Surveillance Program, 2008-2009. Antimicrob Agents Chemother. 2011;55:561-566. doi:10.1128/AAC.01079-10

48. Pfaller MA, Moet GJ, Messer SA, Jones RN, Castanheira M. Geographic variations in species distribution and echinocandin and azole antifungal resistance rates among Candida bloodstream infection isolates: report from the SENTRY Antimicrobial Surveillance Program (2008 to 2009). J Clin Microbiol. 2011;49:396-399. doi:10.1128/JCM.01398-10

49. Fernandez-Ruiz M, Aguado JM, Almirante B, et al. Initial use of echinocandins does not negatively influence outcome in Candida parapsilosis bloodstream infection: a propensity score analysis. Clin Infect Dis. 2014;58:1413-1421. doi:10.1093/cid/ciu158

50. Mora-Duarte J, Betts R, Rotstein C, et al. Comparison of caspofungin and amphotericin B for invasive candidiasis. $N$ Engl J Med. 2002;347 (347):2020-2029. doi:10.1056/NEJMoa021585

51. Kuse ER, Chetchotisakd P, da Cunha CA, et al. Micafungin versus liposomal Amphotericin B for candidaemia and invasive candidosis: a phase III randomised double-blind trial. Lancet. 2007;369:1519-1527. doi:10.1016/S0140-6736(07)60605-9

52. Ullmann AJ, Sanz MA, Tramarin A, et al. Prospective study of amphotericin B formulations in immunocompromised patients in 4 European countries. Clin Infect Dis. 2006;43(4):e29-38. doi:10.1086/505969

53. Rex JH, Bennett JE, Sugar AM, et al. A randomized trial comparing fluconazole with amphotericin B for the treatment of candidemia in patients without neutropenia. Candidemia Study Group and the National Institute. $N$ Engl J Med. 1994;331(20):1325-1330. doi:10.1056/NEJM199411173312001

54. Anaissie EJ, Vartivarian SE, Abi-Said D, et al. Fluconazole versus amphotericin B in the treatment of hematogenous candidiasis: a matched cohort study. Am J Med. 1996;101(2):170-176. doi:10.1016/S0002-9343(96)80072-6

55. Kullberg BJ, Sobel JD, Ruhnke M, et al. Voriconazole versus a regimen of amphotericin B followed by fluconazole for candidaemia in non-neutropenic patients: a randomised non-inferiority trial. Lancet. 2005;366(9495):1435-1442. doi:10.1016/S0140-6736(05) 67490-9

56. Pappas PG, Rotstein CM, Betts RF, et al. Micafungin versus caspofungin for treatment of candidemia and other forms of invasive candidiasis. Clin Infect Dis. 2007;45(7):883-893. doi:10.1086/ 520980

57. Seiler GT, Ostrosky-Zeichner L. Investigational agents for the treatment of resistant yeasts and molds. Curr Fungal Infect Rep. 2021;1-12. doi:10.1007/s12281-021-00419-5

58. Gamal A, Chu S, McCormick TS, Borroto-Esoda K, Angulo D, Ghannoum MA. Ibrexafungerp, a novel oral triterpenoid antifungal in development: overview of antifungal activity against Candida glabrata. Front Cell Infect Microbiol. 2021;11:642358. doi:10.3389/ fcimb.2021.642358

59. Schwebke JR, Sobel R, Gersten JK, et al. Ibrexafungerp versus placebo for vulvovaginal candidiasis treatment: a Phase 3, randomized, controlled superiority trial (VANISH 303). Clin Infect Dis;2021. ciab750. doi:10.1093/cid/ciab750

60. Mesquida A, Vicente T, Reigadas E, et al. In vitro activity of ibrexafungerp and comparators against Candida albicans genotypes from vaginal samples and blood cultures. Clin Microbiol Infect. 2021;27 (6):915.e5-915.e8. doi:10.1016/j.cmi.2021.02.006

61. Arendrup MC, Jørgensen KM, Hare RK, Chowdhary A. In Vitro activity of ibrexafungerp (SCY-078) against Candida auris isolates as determined by EUCAST Methodology and Comparison with Activity against $C$. albicans and $C$. glabrata and with the activities of six comparator agents. Antimicrob Agents Chemother. 2020;64(3): e02136-19. doi:10.1128/AAC.02136-19 
62. Zhao Y, Perlin DS. Review of the novel echinocandin antifungal rezafungin: animal studies and clinical data. J Fungi. 2020;6 (4):192. doi:10.3390/jof6040192

63. Pfaller MA, Carvalhaes C, Messer SA, Rhomberg PR, Castanheira M. Activity of a long-acting echinocandin, rezafungin, and comparator antifungal agents tested against contemporary invasive fungal isolates (SENTRY Program, 2016 to 2018). Antimicrob Agents Chemother. 2020;64(4):e00099-20. doi:10.1128/ AAC.00099-20

64. Helleberg M, Jørgensen KM, Hare RK, Datcu R, Chowdhary A, Arendrup MC. Rezafungin in Vitro activity against contemporary Nordic clinical Candida isolates and Candida auris determined by the EUCAST Reference Method. Antimicrob Agents Chemother. 2020;64(4):e02438-19. doi:10.1128/AAC.02438-19

65. Thompson GR, Soriano A, Skoutelis A, et al. Rezafungin versus Caspofungin in a phase 2, randomized, double-blind study for the treatment of candidemia and invasive candidiasis- the STRIVE trial. Clin Infect Dis;2020. ciaa1380. doi:10.1093/cid/ciaa1380

66. Brand SR, Degenhardt TP, Person K, et al. A phase 2, randomized, double-blind, placebo-controlled, dose-ranging study to evaluate the efficacy and safety of orally administered VT-1161 in the treatment of recurrent vulvovaginal candidiasis. Am J Obstet Gynecol. 2018;218 (6):624.e1-624.e9. doi:10.1016/j.ajog.2018.03.001

67. Brand SR, Sobel JD, Nyirjesy P, Ghannoum MA, Schotzinger RJ, Degenhardt TP. Randomized phase 2 Study of VT-1161 for the treatment of acute vulvovaginal candidiasis. Clin Infect Dis. 2020;1: ciaa1204.
68. Nishimoto AT, Wiederhold NP, Flowers SA, et al. In vitro activities of the novel investigational tetrazoles VT-1161 and VT-1598 compared to the triazole antifungals against azole-resistant strains and clinical isolates of Candida albicans. Antimicrob Agents Chemother. 2019;63(6):e00341-19.

69. Schell WA, Jones AM, Garvey EP, Hoekstra WJ, Schotzinger RJ, Alexander BD. Fungal CYP51 inhibitors VT-1161 and VT-1129 exhibit strong in vitro activity against Candida glabrata and C. krusei isolates clinically resistant to azole and echinocandin antifungal compounds. Antimicrob Agents Chemother. 2017;61(3): e01817-16. doi:10.1128/AAC.01817-16

70. Shaw KJ, Ibrahim AS. Fosmanogepix: a review of the first-in-class broad spectrum agent for the treatment of invasive fungal infections. J Fungi. 2020;6(4):239. doi:10.3390/jof6040239

71. Pfaller MA, Huband MD, Flamm RK, Bien PA, Castanheira M. Antimicrobial activity of manogepix, a first-in-class antifungal, and comparator agents tested against contemporary invasive fungal isolates from an international surveillance programme (2018-2019). J Glob Antimicrob Resist. 2021;26:117-127. doi:10.1016/j. jgar.2021.04.012

72. Arendrup MC, Jørgensen KM. Manogepix (APX001A) displays potent in vitro activity against human pathogenic yeast, but with an unexpected correlation to fluconazole MICs. Antimicrob Agents Chemother. 2020;64(7):e00429-20. doi:10.1128/AAC.00429-20
Infection and Drug Resistance

\section{Publish your work in this journal}

Infection and Drug Resistance is an international, peer-reviewed openaccess journal that focuses on the optimal treatment of infection (bacterial, fungal and viral) and the development and institution of preventive strategies to minimize the development and spread of resistance. The journal is specifically concerned with the epidemiology of

\section{Dovepress}

antibiotic resistance and the mechanisms of resistance development and diffusion in both hospitals and the community. The manuscript management system is completely online and includes a very quick and fair peerreview system, which is all easy to use. Visit http://www.dovepress.com/ testimonials.php to read real quotes from published authors. 